Décadrages Décadrages

cınéma, à travers champs Cinéma, à travers champs

3 | 2004

Hitchcock côté cour

\title{
Fenêtre sur cour, la petite lucarne et l'Amérique des années 50 : notes sur la construction du (de la) téléspectateur(trice)
}

Mireille Berton

\section{(2) OpenEdition \\ Journals}

Édition électronique

URL : http://journals.openedition.org/decadrages/556

DOI : $10.4000 /$ decadrages.556

ISSN : 2297-5977

Éditeur

Association Décadrages

Édition imprimée

Date de publication : 10 avril 2004

Pagination : $59-72$

ISBN : 978-2-9700582-0-5

ISSN : 2235-7823

Référence électronique

Mireille Berton, « Fenêtre sur cour, la petite lucarne et l'Amérique des années 50 : notes sur la construction du (de la) téléspectateur(trice) », Décadrages [En ligne], 3 | 2004, mis en ligne le 29 avril 2013, consulté le 19 avril 2019. URL : http://journals.openedition.org/decadrages/556 ; DOI : 10.4000/ decadrages.556 


\section{Fenêtre sur cour, la petite lucarne et l'Amérique \\ des années 50 : notes sur la construction du (de}

\section{la) téléspectateur(trice)}

\author{
par Mireille Berton
}

La tradition critique qui s'est élaborée autour de Fenêtre sur cour interprète le plus souvent cette œuvre comme une métaphorisation de l'expérience du spectateur cinématographique. Il s'agit en effet de souligner la nature auto-réflexive d'un film qui montre Jefferies épiant (dans l'ombre, donc sans être vu) ses voisins habitant de l'autre côté d'une cour intérieure. Conformément à cette lecture, la fenêtre du héros - mais aussi celles qui lui font face - fonctionnerait comme un écran cinématographique gratifiant Jefferies, confortablement assis sur un fauteuil (roulant), d'une série de "spectacles» comprenant les scènes les plus coutumières (se lever, danser, se raser, se restaurer, se disputer, travailler, etc.), mais aussi les plus insolites (se débarrasser de sa femme, d'un chien)1. Teintant leurs études d'un zest de psychanalyse, certains auteurs conçoivent ces fenêtres-écrans comme des supports servant à la projection des désirs et angoisses du héros sur sa vie professionnelle et sentimentale 2 .

A l'étude de cette composante méta-cinématographique s'ajoute celle du voyeurisme caractérisant la position d'un personnage animé par un intense désir de voir qui exige, toutefois, le maintien d'une distance entre l'organe générateur, l'œil comme source pulsionnelle, et l'objet regardé. Dans l'historiographie du film, le voyeurisme semble être un des aspects le plus fréquemment commenté, qu'il soit appréhendé comme une critique (ou non) de Hitchcock à l'égard d'une "pathologie" qui gagne une société aux prises avec les contradictions d'une culture de masse en pleine expansion. Selon la plupart des commentateurs, c'est à l'analyse de Jean Douchet que remonterait la mise à jour de ce regard voyeuriste propre au spectateur cinématographique ${ }^{3}$. Cette exégèse du film comme symbolisant l'activité spectatorielle, mais aussi la nature du plaisir cinématographique, est d'ailleurs corroborée par le cinéaste lui-même qui, dans son célèbre entretien avec Truffaut, met en exergue
1 L'analogie de la fenêtre avec l'écran cinématographique apparaît chez de nombreux auteurs. Voir, par exemple, Alfred Hitchcock's Rear Window, John Belton (éd.), Cambridge University Press, Cambridge, 2000 ou John Fawell, Hitchcock's Rear Window, Southern Illinois University Press, Carbondale/Edwardsville, 2001.

2 Nombre d'études contemporaines prouvent la pérennité de ce genre d'interprétation. Lire, par exemple, John A. Bertolini, "Rear Window, or the Reciprocated Glance", Framing Hitchcock. Selected Essays from the Hitchcock Annual, Christopher Brookhouse et Sidney Gottlieb (éd), Wayne State University Press, Detroit, 2002, p. 234-250.

3 Jean Douchet, "Hitch et son public", in Cahiers du Cinéma, no 113, nov. 1960, p. 7-15. II pense, en effet, que "c'est là qu'Hitchcock expose sa conception même du cinéma (c'est le cinéma dans le cinéma), révèle ses secrets, dévoile ses intentions. James Stewart, reporter photographe, y est avant tout un spectateur. C'est l'une des raisons pour lesquelles on le voit lié à son fauteuil. A travers lui, Hitchcock entend définir la nature du spectateur et, particulièrement, la nature du spectateur hitchcockien. Ce dernier est un voyeur. II veut jouir du spectacle. Ce qu'il regarde sur l'écran (donc, ce que Stewart regarde dans l'immeuble de l'autre côté de la cour), c'est la propre projection de lui-même. Elle seule, a priori, est capable de l'intéresser. D'une façon ou d'une autre, c'est lui-même qu'il vient voir " (p. 8). 
4 II reconnaît également, dans cette même interview, qu'il s'agit d'un film sur le cinéma ou d'un film "purement cinématographique". "Vous avez, dit-il, l'homme immobile qui regarde au-dehors. C'est le premier morceau de film. Le deuxième morceau fait apparaître ce qu'il voit et le troisième montre sa réaction. Cela représente ce que nous connaissons comme la plus pure expression de l'idée cinématographique". Hitchcock/Truffaut, François Truffaut (éd.), Editions Ramsay, Paris, 1983, p. 178.

5 "Un homme regarde et attend pendant que nous regardons cet homme et attendons ce qu'il attend. [...] Constamment, nous nous dédoublons, tandis que le héros du film se dédouble, nous identifiant à lui, pendant qu'il s'identifie à l'homme qu'il épie". Claude Chabrol et Eric Rohmer, Hitchcock, Editions Universitaires, Paris, 1957, p. 126-127.

6 Remarquons au passage que j'exclue volontairement le "pendant narratologique" de cette lecture métapsychologique de Fenêtre sur cour. En effet, David Bordwell, dans son ouvrage sur la narration du film de fiction, a analysé la manière dont le film décrit le travail effectué par le spectateur face à un film dont il s'agit de comprendre et d'interpréter la trame narrative. Comme Jeff assis dans son fauteuil, tout spectateur est amené au cours d'un film à formuler, sur la base de certaines informations, des hypothèses, des déductions, des inférences et des attentes qui lui permettent de construire une certaine version de l'histoire racontée par l'instance énonciatrice. Le film offrirait ainsi une sorte de modèle réduit de l'expérience spectatorielle en termes de compréhension narrative car, comme tout film de fiction, Fenêtre sur cour exige du spectateur d'être attentif à toutes sortes d'éléments (visuels ou non) donnant la clé du mystère (ici du meurtre) ou de l'intrigue. David Bordwell, Narration in the Fiction Film, University of Wisconsin Press, Madison, 1985, p. 29-47.

7 Tania Modleski, Hitchcock et la théorie féministe. Les femmes qui en savaient trop, L'Harmattan, Paris, 2002 [1988, Methuen]. Lire à ce propos son introduction, "Hitchcock, le féminisme et l'inconscient patriarcal", p. 9-31.

8 Je pense surtout à John Fawell qui consacre deux paragraphes à cette question dans son Hitchcock's Rear Window, op. cit., p. 130. Mais citons aussi Francis Montcoffe qui en dit deux mots dans son petit livre consacré au film: "On peut aussi y distinguer une certaine amertume, non pas tant due aux 'vices, de l'humanité, le profil fondamentalement voyeuriste de son protagoniste ${ }^{4}$. Dans un ouvrage antérieur à l'article de Douchet, toutefois, Rohmer et Chabrol signalent déjà que la «théorie du spectacle» élaborée par le film permet de traiter du "thème [qui] concerne l'essence même du cinéma": la pulsion scopique ou la passion du voir ${ }^{5}$.

Personne donc ne semble contester l'idée que Fenêtre sur cour donne une définition «canonique» du spectacle cinématographique à travers la mise en place d'un dispositif de vision semblable au cinéma ${ }^{6}$. La théorie féministe elle-même, malgré ses tentatives visant à remettre en question la suprématie du regard masculin à l'œuvre dans ce même dispositif, semble reconduire ce consensus critique en incluant les spectatrices dans ce modèle interprétatif $\mathbf{7}$. Si cette unanimité me paraît amplement justifiée, il me semble cependant que cette idée du film comme mise en abîme dérive de la perspective auteuriste adoptée par des critiques qui s'emploient à exalter une conception hitchcockienne du cinéma fondamentalement méta-discursive et hautement représentative du travail de "manipulation» exercé par tout film de fiction à l'endroit du spectateur. Cette focalisation sur le texte filmique comme reflétant la pensée de l'auteur sur le cinéma a comme conséquence directe l'occultation relative des conditions de production de Fenêtre sur cour, ainsi que du contexte historique et socioculturel dans lequel il s'inscrit. En effet, la définition du rôle du spectateur fournie par le film mérite, selon moi, d'être confrontée à des données extra-filmiques et extra-cinématographiques qui dépassent largement les limites narratives de l'intrigue et les intentions «manifestes» de Hitchcock.

\section{Cinéma et télévision dans les années $\mathbf{5 0}$ aux Etats-Unis}

Je souhaiterais donc aborder un aspect rarement évoqué à propos de ce film ${ }^{8}$, à savoir ses relations avec un médium de masse qui commence à gêner l'industrie du cinéma en proie à une crise sans précédent - la télévision - ainsi qu'avec la culture de masse et la société de consommation américaines qui fleurissent dans les années 50 . Il me paraît en effet intéressant de remarquer que Fenêtre sur cour déplie toute une réflexion sur le cinéma au moment même où la télévision vient bouleverser le paysage audiovisuel et redéfinir la position du spectateur face à une série d'images en mouvement. Dès 1950, la désertion croissante des salles de cinéma, délaissées au profit de la télévision, pousse Hollywood à lancer toutes sortes de procédés d'écrans larges destinés à épater le public et à le submerger de sensations et d'émotions inédites 9 . Tourné et distribué en format Vistavision $\mathbf{1 0}$, Fenêtre sur cour semble donc répondre à cette stratégie de compétitivité voulue par les Majors américaines qui, après 
avoir échoué dans leur tentative d'assimilation du marché télévisuel11, doivent se résoudre à lutter avec leurs propres armes en exploitant les spécificités techniques du cinéma. Il me semble pourtant que si le film participe historiquement à ce climat de défiance du grand écran à l'égard du petit, il entretient avec cette dichotomie cinéma-TV un rapport autrement plus complexe et articulé.

En effet, plusieurs éléments permettent d'opérer une lecture différente du dispositif visuel formé par le décor du film. Le parallèle habituellement tiré entre le personnage de Jeff et le spectateur de cinéma peut aisément être étendu, à mon avis, au téléspectateur engagé dans un régime scopique très proche de celui qui est expérimenté par le protagoniste. Installé en position semi-couchée, dans une quasi-immobilité, face à une série de fenêtres qui ressemblent plus à des écrans de télévision qu’à des écrans de cinéma ${ }^{\mathbf{1 2}}$, le photographe aime à tromper son ennui en regardant les péripéties de la vie d'autrui, épisodes devant lesquels il lui arrive, parfois, de manger ou de s'assoupir. Tout concourt à faire de Jeff un téléspectateur type, happé par un spectacle banal - donc peu cinématographique (hormis le crime auquel, cependant, il n'a pas assisté) - qu'il appréhende sous la forme fragmentaire d'un zapping, passant d'une fenêtre-écran à l'autre pour suivre les bribes de différentes histoires. Un bémol toutefois à ce parallèle puisque ce n'est qu'à partir de l'invention de la télécommande, à la fin des années 70, que la pratique du zapping a été rendue possible ${ }^{\mathbf{1 3}}$. Si cette référence à la télévision est très rarement relevée, je tenterai ici d'étayer plus longuement cette hypothèse en revenant sur la période d'émergence de la télévision comme médium de masse entrant en concurrence directe avec une forme éprouvée de consommation d'images. Je compléterai ce point de vue en prenant en considération que cette période d'après-guerre correspond également à un moment de l'histoire des Etats-Unis où s'élabore un modèle de vie sociale et familiale basé sur une distribution claire des rôles en termes d'identité sexuelle. Nous verrons comment l'idéologie dominante véhiculée par les médias, et notamment par des programmes télévisés et la publicité, apparaît retravaillée dans le film de Hitchcock sous une forme à la fois originale et ambivalente. Il s'agira donc d'évoquer les différentes fonctions de la télévision dans le cadre d'une société de consommation qui suscite des débats sans fin sur les effets de cette boîte à images jugée responsable, pour certains, de mettre en péril l'ensemble de la culture dite noble, ainsi que les modèles identitaires.

Par conséquent, si Fenêtre sur cour participe indéniablement à l'histoire et la théorie du cinéma, il contribue également, en déployant un dispositif de type "télévisuel», à une histoire culturelle qui exige la qu'à ce ' vice, nouveau de l'Amérique que représente la télévision et son intrusion obsédante dans le quotidien: on regarde toute la journée, on attend passivement l'événement, on veut qu'il se produise, on accepte des spectateurs étriqués et morcelés, etc.". Cf. F. Montcoffe, Fenêtre sur cour, Nathan, coll. Synopsis, Paris, 1990, p. 19.

9 II s'agit notamment de procédés d'anamorphose qui permettent d'élargir l'image de l'écran comme le Cinémascope (2.55:1), introduit en 1953, ou le Scope (2.35:1).

10 Procédé lancé par la Paramount, productrice du film, en 1954. II n'est pas sûr que le film ait toujours été vu sous ce format car peu de salles s'équipèrent pour la projection d'un tel procédé fondé sur le déroulement horizontal du film $35 \mathrm{~mm}$ traditionnel, et ceci en raison des coûts engendrés par une telle installation.

11 Après la Seconde Guerre mondiale, les Etats-Unis sont le premier pays à se lancer dans l'industrie de la télévision, notamment en créant des "salles de télévision" qui diffusent sur grand écran des programmes à un public ayant payé sa place, comme au cinéma. Mais Hollywood, au moment où les ventes de postes de télévision augmentent considérablement, ne parviendra pas imposer la télévision en tant que spectacle et divertissement collectifs. Notons au passage que c'est précisément la Paramount - productrice de Fenêtre sur cour - qui dès 1947-48 organisera des transmissions en direct de grands événements sportifs ou politiques dans des salles de cinéma. Par ailleurs, l'industrie cinématographique sera pendant longtemps exclue des opérations financières réalisées par les télévisions commerciales. Lire à ce propos Peter Kramer, "The Lure of the Big Picture: Film, Television and Hollywood", Big Picture, Small Screen. The Relations between Film and Television, John Hill et Martin McLoone (éd.), University of Luton Press, Luton, 1996, p. 9-46.

12 Le format des fenêtres est en fait variable puisque si certaines sont carrées, comme le format des films muets ou l'écran de télévision, celles qui se situent au centre du champ de vision de Jeff, comme la fenêtre des Thorwald, adoptent des proportions semblables à celles du Vistavision. Le décor combine donc des écrans TV et cinématographiques.

13 Le passage d'un programme à l'autre est une pratique qui est d'abord apparue aux Etats-Unis, au moment de la diffusion des publicités. Cf. Hélène Duccini, La télévision et ses mises en scène, Paris, Nathan, 1998. 


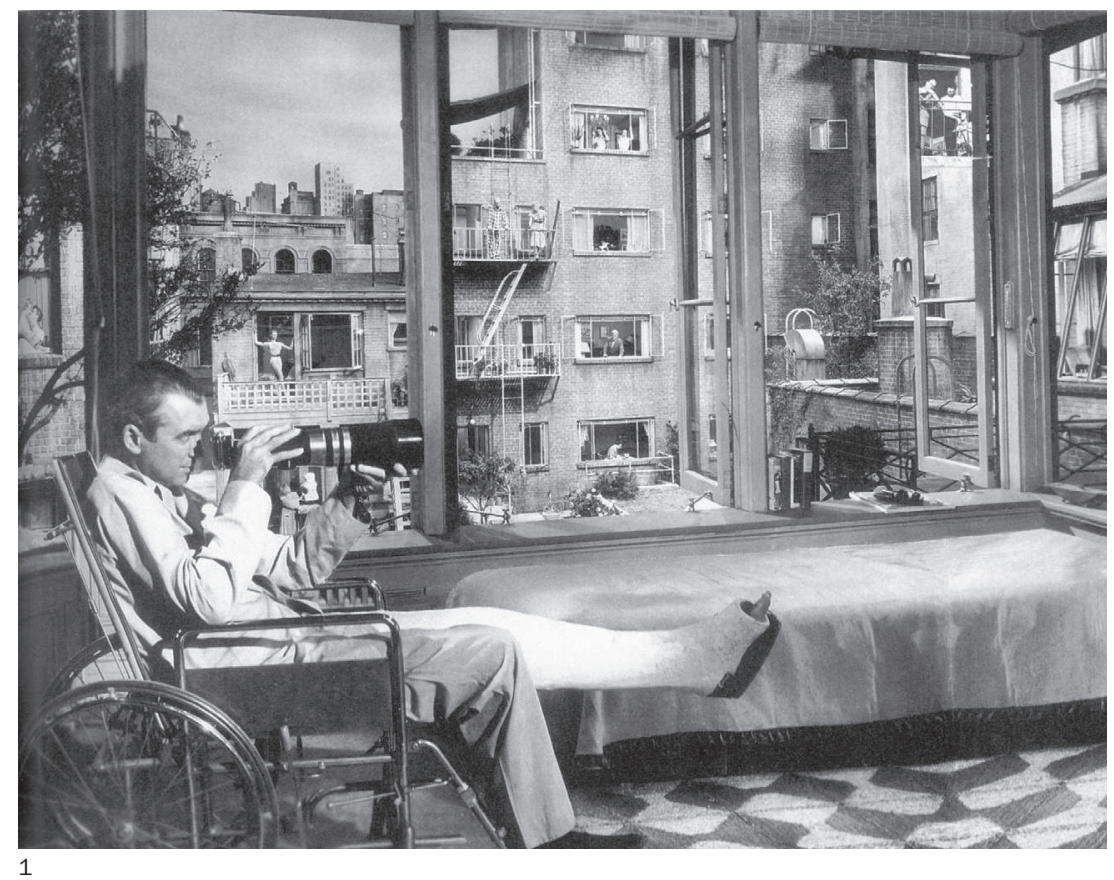

14 Au début des années 50, l'industrie cinématographique, qui se voit refuser la possibilité de racheter l'industrie télévisuelle, doit se contenter de lui fournir des produits comme des séries ou des films. Cette mise à l'écart d'un marché financier lucratif provoque une frustration que les studios tentent d' "exorciser" sous la forme de productions de films qui sont autant de tracts anti-télévision. Ainsi, le déclin du cinéma va contribuer à la réalisation de films qui dépeignent la télévision comme un instrument bassement mercantile responsable d'abêtir et de corrompre moralement le public. Lire à ce sujet, Jane Stokes, On Screen. Cinema and Television in the United States and Britain, MacMillan Press, Houndsmills/Londres, 1999. prise en considération de paramètres à la fois sociaux, historiques et idéologiques. Les rapports entre télévision et cinéma à l'œuvre dans ce film seront analysés à deux niveaux distincts : à un niveau "structurel», je reviendrai sur la représentation du dispositif visuel et la position du (télé)spectateur dans les années 50, pour ensuite aborder cette problématique à un niveau narratif en envisageant le contenu des images diffusées par les fenêtres-écrans et leurs liens avec un certain type d'émissions TV - les soap opera - centrées sur des thèmes similaires comme le mariage, le couple, la famille. J'espère montrer, par ce biais, que Fenêtre sur cour, malgré la "guerre» que se livrent le cinéma et la télévision, en dit plus sur cette dernière qu'un grand nombre de films hollywoodiens de cette époque tournant en dérision un médium qui est parvenu à développer une identité culturelle indépendante14.

Fenêtre sur cour sort en 1954, année décisive pour Hollywood qui doit, à la fois, reconsidérer ses volontés d'hégémonie en matière de divertissement de masse et miser sur une série d'expédients destinés à remplir les salles de cinéma, comme par exemple les grands formats, la systématisation de la couleur, le renforcement du star system ou la production de films à grand spectacle. Alors que pendant la Seconde Guerre mondiale les cinémas aux Etats-Unis comptabilisaient environ 
8 millions d'entrées par semaine, en 1954 ce chiffre a chuté à moins de 4 millions $\mathbf{1 5}$. Cette année-là s'avère par contre florissante pour l'industrie télévisuelle dont les ventes de postes de télévision explosent: de 4 millions de récepteurs en service en 1950, on passe à plus de 20 millions en 1954. Dès 1953, les programmes télévisés diffusés par plusieurs chaînes sont regardés quotidiennement par 80 millions d'Américains qui considèrent désormais la télévision comme un appareil domestique aussi courant que la machine à laver ou l'aspirateur. S'il faudra attendre 1968 pour que plus de 90\% des foyers américains soient équipés, en 1954 la TV est complètement entrée dans les mœurs en tant que mass media, mais aussi comme instrument informatif et récréatif à usage privé16.

\section{Le dispositif visuel dans Fenêtre sur cour: le principe du multi-écrans}

Présenté comme un photographe reporter en convalescence, le héros de Fenêtre sur cour, visiblement désœuvré, observe ses voisins en position de "voyeur couché» $\mathbf{1 7}$ à travers l'encadrement formé par les fenêtres des appartements (fig. 1). Au fil de ces séances rituelles rythmant sa journée, il finit par se familiariser avec chacune de ces personnes, c'est-à-dire avec leurs histoires personnelle et affective. La captation de ces microrécits à des intervalles plus ou moins réguliers, ainsi que le glissement d'une "série diégétique» à l'autre, s'apparentent à une pratique courante à l'époque, celle de la consommation de programmes télévisuels composés surtout de shows, de feuilletons et de films. Le gigantesque décor constitué de 4 façades, de 31 appartements (vus depuis la fenêtre de Jeff), dont 12 entièrement meublés, fournit un dispositif favorisant une perception à la fois englobante et diffractée puisque le héros a la possibilité de passer librement d'un "écran» à l'autre, à l'instar du téléspectateur qui zappe d'une chaîne à l'autre au moment voulu. Face à Jeff, donc, se déploie un mur d'écrans qui lui permet de choisir librement le "programme» ou l'épisode de la série (Mlle Torse (fig. 2), Mlle Cœur brisé, Les Jeunes mariés, Le Mariage raté, Le Musicien solitaire (fig. 3), etc.) qu'il souhaite regarder, à cette différence près que si la télévision présente des fragments d'univers de manière diachronique, dans Fenêtre sur cour, ils peuvent être (théoriquement) embrassés d'un seul regard, donc appréhendés dans la synchronie. Le générique inaugure d'ailleurs ce principe de surcadrage puisqu'il se déroule sur le fond d'une fenêtre divisée en trois battants dont les rideaux en bambou respectifs se lèvent progressivement pour dévoiler le lieu du drame à venir: la cour à son tour morcelée en différentes zones découpant l'espace habitable18.

Si le décor de Fenêtre sur cour inscrit en son sein une logique perceptive de type télévisuel, il rappelle tout autant les dispositifs multi-écrans
15 Peter Kramer, "The Lure of the Big Picture: Film, Television and Hollywood", op. cit.

16 Chiffres tirés de Pierre Miquel, Histoire de la radio et de la télévision, Éditions Richelieu, Paris, 1972.

17 J'emprunte cette expression de "position du voyeur couché" à François Albera qui analyse dans un article les liens entre Hitchcock et Klossowski. Cette position correspond, selon moi, à la position même du téléspectateur qui, affalé sur le canapé ou couché dans son lit, a perdu la rectitude et la semi-verticalité imposées par les fauteuils de cinéma, autant que par les règles de bienséance. En effet, à l'abri des regards, une certaine latitude est permise au téléspectateur qui peut alors relâcher son corps à sa guise. $C f$. F. Albera, "La position du voyeur couché. Hitchcock avec Klossowski", in Cinémathèque, $\mathrm{n}^{\circ} 13$, printemps 1998 , p. 91-100.

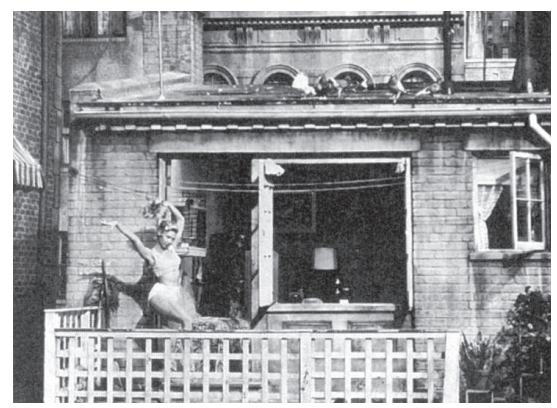

2

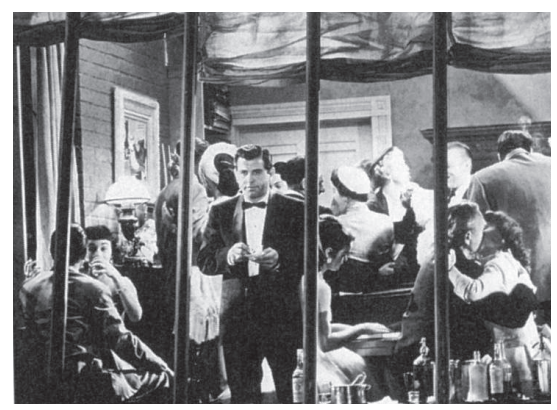

3

18 Pour une étude sociologique et architecturale du décor du film, lire James Sanders, "Worlds of Differences. Dead End and Rear Window", Celluloid Skyline: New York and the Movies, Alfred A. Knopf, New York, 2001, p. 219-241. 
19 Ce procédé, qui symbolise certainement aussi la frénésie schizophrénique caractérisant cette pulsion du tout voir et du tout entendre propre à notre société, trouve logiquement un terrain d'application idéal à la télévision qui réalise à foison des émissions ou séries jouant sur le principe de l'écran divisé. Les studios sont d'ailleurs toujours organisés autour de moniteurs renvoyant des images du plateau, dispositif qui est parfois repris de manière redondante par les journaux télévisés usant d'un fond tapissé d'écrans. Remarquons que si la technique de l'écran multiple (ou démultiplié) est devenue un élément spécifique du média TV, le cinéma depuis ses origines connaît la méthode du "split screen" permettant de montrer en simultané deux ou plusieurs lignes d'actions parallèles.

$20 \mathrm{~A}$ ce sujet et pour la suite de mon argumentation, je m'appuie sur l'article de Beatriz Colomina, "Enclosed by Images: Architecture in the Post-Sputnik Age", CTRL Space. Rhetorics of Surveillance from Bentham to Big Brother, Ursula Frohne, Thomas Y. Levin et Peter Weibel (éd.), ZKM-Center for Art and Media / The MIT Press, Karlsruhe / Londres, 2002, p. 323-337.

21 Parmi leurs œuvres les plus représentatives, citons le film Glimpses of USA projeté sur 7 écrans suspendus à l'intérieur du dôme réalisé à l'occasion de l'Exposition américaine de Moscou en 1959. Le film consiste en un long zoom avant allant de vues sur l'espace cosmique vers des images d'espaces domestiques représentant la vie quotidienne de l'Américain moyen. Voir Beatriz Colomina, op. cit.

DISPOSITIF DE PROJECTION DU FILM GLIMPSES OF THE USA DE CHARLES ET RAY EAMES, RÉALISÉ À L'OCCASION DE L'EXPOSITION AMÉRICAINE DE MOSCOU EN 1959 aujourd'hui couramment utilisés dans différents contextes, et notamment à la télévision qui les a institués en véritables topoi connotant la simultanéité et la toute-puissance des transmissions en direct19. En 1954, cependant, ce motif semble renvoyer à un réseau référentiel supplémentaire, puisque cette structure d'écrans multiples trouve son origine dans le développement des technologies militaires. En effet, la juxtaposition d'images animées et la présentation d'informations concomitantes organisaient déjà le fonctionnement des Situations Rooms, lieux de réception d'images, de présentation et d'analyse des opérations militaires durant la Seconde Guerre mondiale. Par la suite, des artistes, graphistes et architectes américains se sont inspirés de ce modèle pour le mettre en ouvre dans le cadre d'expositions, de performances ou de structures architecturales fixes $\mathbf{2 0}$.

Les designers Charles et Ray Eames sont connus pour avoir exploité, en particulier durant les années 50 et 60 , un mode de perception fondé sur une mosaïque d'images provoquant une surabondance de stimuli sensoriels (fig. 4). Réalisant pour leurs expositions multi-médias des films qu'ils projettent sur plusieurs écrans, ils cherchent à définir ces espaces didactiques conformément aux nouvelles formes de communication de masse telles que la télévision, l'imagerie spatiale ou l'industrie informatique 21. Il s'agit à chaque fois de mettre en jeu une logique particulière de transmission des informations qui s'effectue sur le mode d'un flux à la fois multiple et discontinu d'images qui finit par excéder

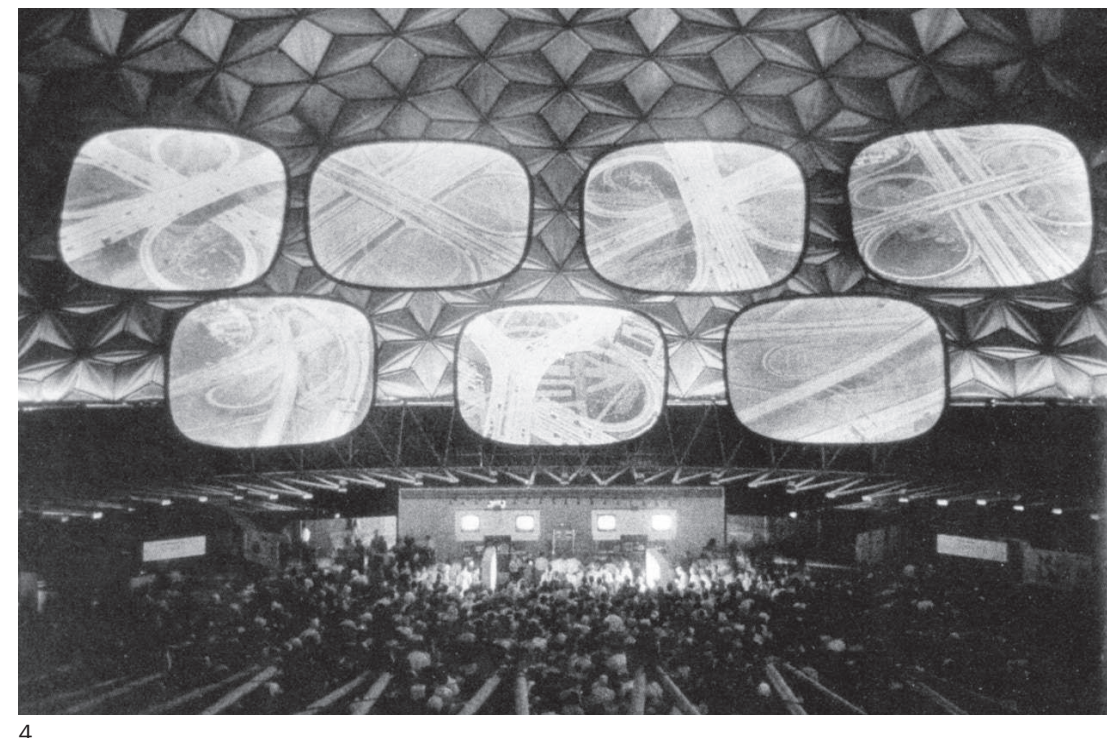


la capacité d'absorption du visiteur-spectateur. Les dispositifs multiécrans des Eames, en invalidant une lecture linéaire et univoque de l'espace, provoquent une désorientation à laquelle le lecteur, le téléspectateur et le consommateur des années 50 sont déjà confrontés dans leur vie quotidienne lorsqu'ils ouvrent un journal, allument leur télévision ou se rendent au supermarché. Ces mosaïques d'images symbolisent ainsi cette nouvelle société de communication qui combine différentes sources d'information, marquant ainsi le début de nouvelles modalités perceptives qui articulent de manière inédite les notions de distraction et de concentration $\mathbf{2 2}$.

En 1954, les spectateurs de Fenêtre sur cour ne sont donc pas étrangers à un mode de perception fragmenté et discontinu qui les oblige à circuler dans un champ de vision superposant différentes données hétéroclites, puisque les mass media eux-mêmes, et la télévision en particulier, opèrent déjà suivant ces règles. Si ce genre de dispositif donne l'illusion

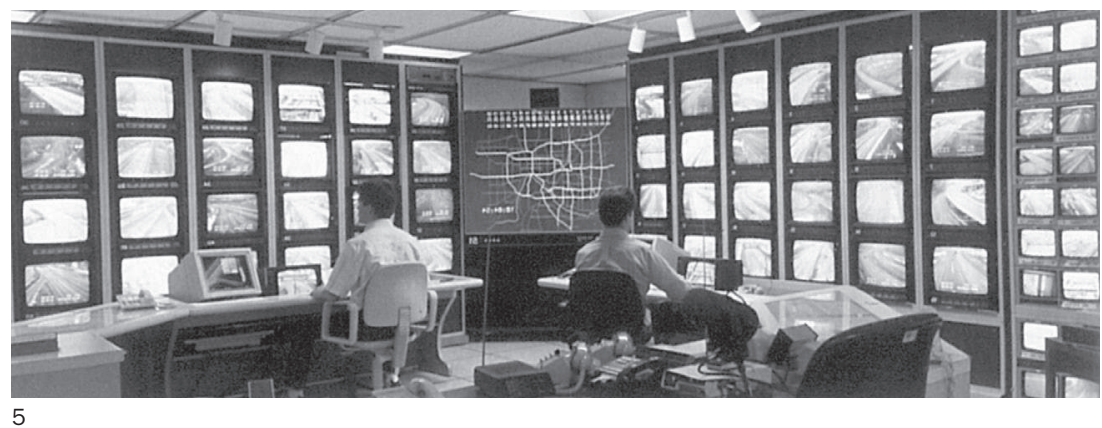

d'une certaine liberté d'action, il dissimule également une idéologie renvoyant au concept de maîtrise du perçu, à partir duquel les différentes formes de surveillance sociale, plus tard, procèderont 23 (fig. 5). De par sa position surplombante, Jeff croit lui aussi bénéficier d'une sorte de toute-puissance perceptive qui lui ménage l'accès à une position privilégiée de témoin d'un crime, ou du moins de ses conséquences directes. Depuis sa fenêtre, son regard tend à adopter une vision omnisciente sur cette arrière-cour structurée en différentes «scènes» désarticulées, mais auxquelles il confère une cohérence en subsumant cet agrégat d'images à son seul point de vue. Je laisse ici le soin au lecteur de tirer les parallèles qui s’imposent entre l'activité de Jeff et celle du téléspectateur amené à jongler continuellement entre différents niveaux de réalité appréhendés dans le morcellement et la dispersion, mais aussi dans l'illusion d'une maîtrise totalisant les données perçues $\mathbf{2 4}$.
22 Durant ces années de guerre froide, toutefois, ces techniques de juxtaposition d'images véhiculent également une idéologie défendue par le président Nixon lui-même qui aime à vanter la supériorité du modèle de vie américain basé sur une forme de liberté inexistante dans les pays communistes. En effet, la société de consommation qui s'épanouit au début des années 50 offre au citoyen américain toute une gamme de produits et de marques qui accrédite sa liberté de choix et flatte son pouvoir d'achat. A cette époque donc, les Russes n'envient pas le système politique américain, mais bien la panoplie d'appareils ménagers, de télévisions et de maisons en banlieue à la portée des classes moyennes et supérieures. Voir Beatriz Colomina, op. cit.

DISPOSITIF DE CONTRÔLE DU MÉTRO DE MINNEAPOLIS, 1993

23 Lire à ce propos, Surveillance, Closed Circuit Television and Social Control, Clive Norris, Jade Moran et Gary Armstrong (éd.), Ashgate, Aldershot, 1998.

24 J'invite le lecteur à approfondir cette brève analyse des connexions entre télévision et Fenêtre sur cour en lisant (ou si cela est déjà fait, en jaugeant l'intertextualité interne à la fois à l'histoire du cinéma et aux articles de cette revue) l'étude d'Alain Boillat sur Brian de Palma qui démontre combien l'esthétique télévisuelle, en "latence" chez Hitchcock, peut être extrapolée par un cinéaste contemporain de manière à la fois révérencieuse et indépendante à l'égard de son modèle. 


\section{Le modèle patriarcal américain et la féminisation du médium télévisuel}

L'expérience de liberté et de contrôle qui caractérise à la fois la situation de Jeff, celle du spectateur inséré dans un dispositif multi-écrans, ainsi que celle du téléspectateur, est rejouée à un autre niveau par la télévision qui homologue, dans les années 50 , un modèle social et familial conforme aux exigences de l'économie de marché libérale et de la politique d'unité nationale. Il s'agit donc ici de s'intéresser aux contenus narratifs des programmes de télévision qui, à cette époque-là, s'adressent au peuple américain par le biais de modèles de consommation et de comportements permettant à chacun de mesurer son adéquation à la norme générale. Sous le caractère apparemment anecdotique de l'intrigue amoureuse qui se noue entre Jeff et Lisa, nous verrons plus loin comment Fenêtre sur cour négocie la question de la différence sexuelle qui préoccupe tant l'Amérique des années 50.

Une des spécificités de cette période réside dans le développement rapide des moyens de communication, dans la diversification croissante des médias et surtout dans l'institutionnalisation de la télévision qui devient le médium d'information et de distraction le plus important. Pour contribuer à l'assimilation et au nivellement de groupes ethniques, de classes sociales ou de schémas identitaires divers, le gouvernement américain mise alors sur une stratégie visant le renforcement du modèle de la famille blanche, de classe moyenne, consumériste, installée dans une maison de banlieue, avec une mère au foyer aimante et parfaite
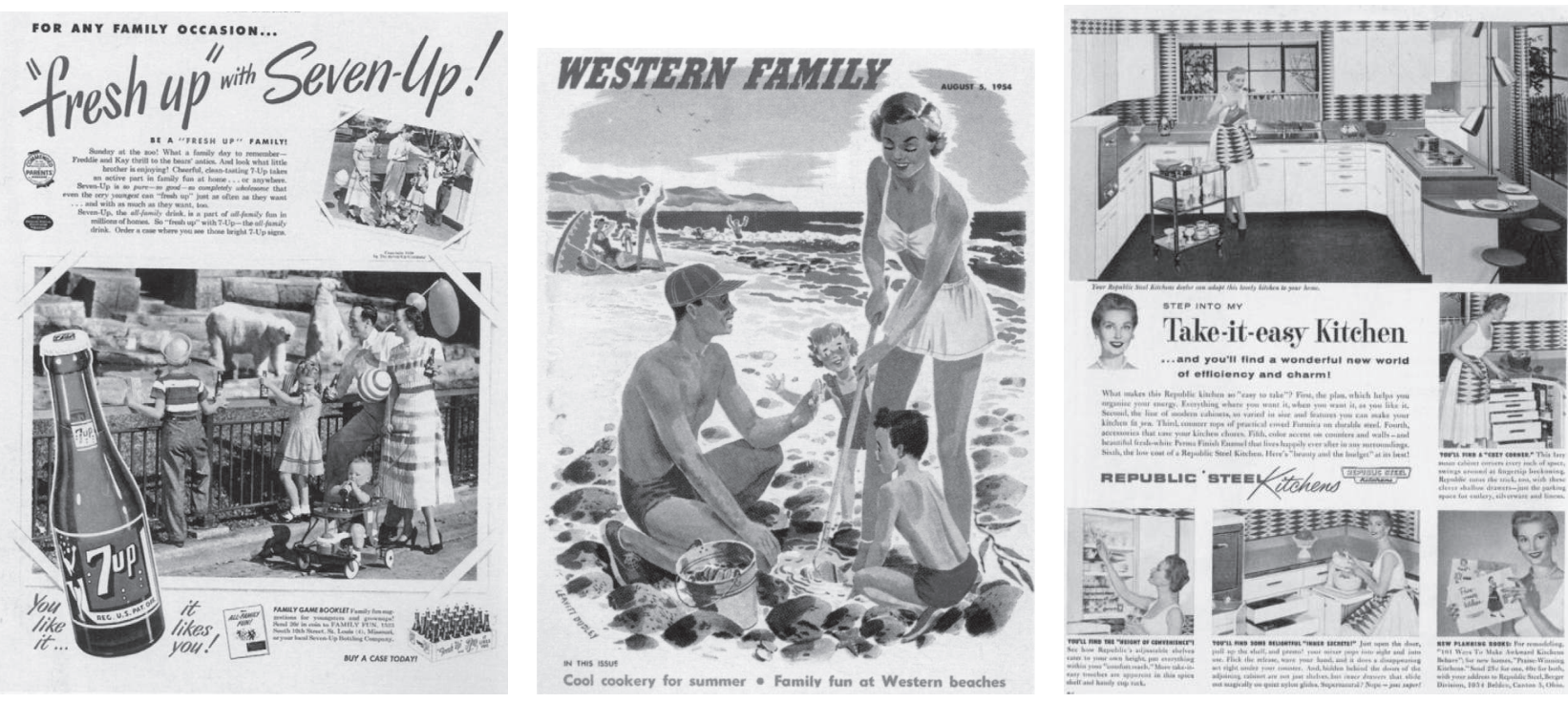
ménagère, ainsi qu'un père travaillant à l'extérieur pour subvenir aux besoins de sa descendance 25 (fig. 6 et 7). Parallèlement, l'expansion de la production industrielle de biens de consommation exige un investissement dans le foyer que l'on équipe en appareils ménagers en tous genres afin de rejoindre le standard moderne du confort matériel (fig. 8). Dans cette entreprise d'homogénéisation nationale fondée sur un idéal commun, la télévision joue un rôle crucial puisqu'en elle se concentrent différents intérêts servant à la fois la société de consommation, l'idéologie de puissance nationale et l'industrie culturelle. Si elle vient à symboliser ce nouvel ordre domestique centré sur le foyer muni de tout l'appareillage technique et des produits nécessaires au bien-être de la famille, comme à la prospérité économique, ses programmes reflètent également un centrement sur la cellule familiale comme modèle de vie. Ce nouvel idéal d'habitation intronise les femmes en tant que gardiennes de l'harmonie communautaire et du bon fonctionnement de l'espace domestique qu'elles ont le devoir de diriger. Ce n'est donc pas par hasard si ce sont elles qui président à l'achat des appareils ménagers, et notamment de la télévision qui devient alors un produit de consommation typiquement féminin 26 (fig. 9 et 10).

Pour mieux comprendre cette association entre télévision et féminité, il est nécessaire de replacer ce syntagme dans le cadre du débat sur les mass media qui mobilise certains critiques américains et européens inquiets du développement d'une société du spectacle jugée responsable

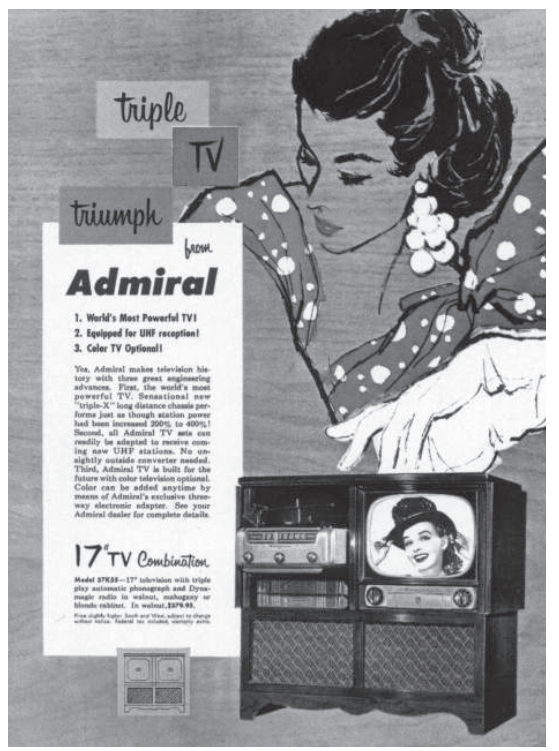

9

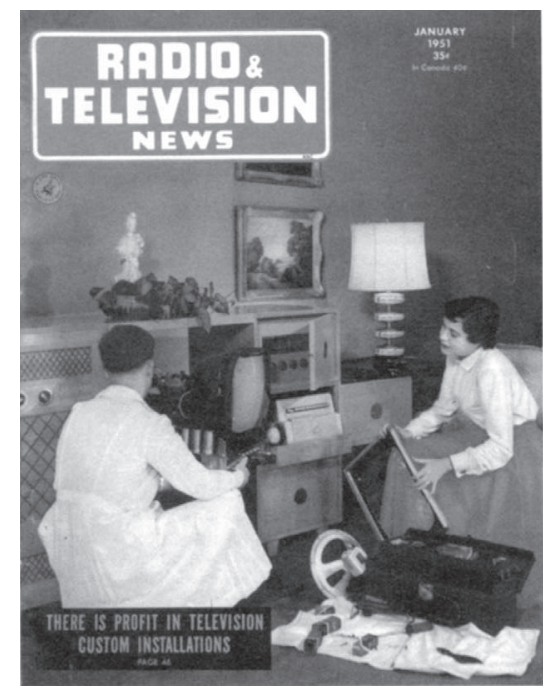

10
25 Les années 50 aux Etats-Unis correspondent à une époque mitigée oscillant entre euphorie économique et angoisses sécuritaires. Après la Seconde Guerre mondiale, émerge dans le discours social un consensus autour de la nécessité d'une identité nationale visant à raffermir la fierté en une communauté unie, stable et homogène. Le boom économique réalisé grâce à l'effort de guerre permet à beaucoup d'Américains de réaliser leur rêve en s'achetant une maison en banlieue, loin du chaos des grandes villes. En 1954, on construit 1,5 million de nouvelles maisons (la plupart en banlieue) qui représenteront le modèle de vie dont les élites politiques et économiques font la propagande.

26 Sur cette question, voir Jane Stokes, On Screen. Cinema and Television in the United States and Britain, op. cit. 
27 Citons les noms des représentants de l'Ecole de Francfort, Adorno et Horkheimer, ainsi que le porte-parole du discours antimass media américain, Dwight Macdonald. Aux Etats-Unis, ce sont des scandales financiers liés à la production de certains shows télévisés qui ont jeté un discrédit durable sur la moralité de l'industrie télévisuelle.

28 Andreas Huyssen, After the Great Divide. Modernism, Mass Culture and Postmodernism, Macmillan, Londres, 1986.

29 La télévision vise alors le public cinématographique qui remplissait traditionnellement les salles de cinéma durant la Seconde Guerre mondiale, c'est-à-dire les femmes. Alors que la guerre ne leur avait pas donné l'occasion de dépenser leur salaire, hormis pour la consommation de films, après le conflit mondial, elles vont investir ces économies dans leur ménage, tout en délaissant les sorties au cinéma.

30 N'ayant pas accès à des informations de première main, je fonde notamment mon argumentation sur l'étude de Kristen Hatch, "Selling Soap. Post-War Television Soap Opera and the American Housewife ", Small Screens, Big Ideas. Television in the 1950s, Janet Thumin (éd.), op. cit., p. 35-49. de banaliser l'art et d'anesthésier l'esprit critique 27. Sous-tendue par une idéologie anti-démocratique, cette critique des mass media jette surtout l'opprobre sur la télévision, accusée de participer activement au processus de déshumanisation opéré par l'industrialisation de la culture de masse. Andreas Huyssen remarque qu'au XIX ${ }^{e}$ siècle déjà, les élites intellectuelles réfèrent la sphère de la culture populaire à la féminité, à une époque où les exigences d'égalité sociale et sexuelle viennent mettre en péril le modèle patriarcal sur lequel repose l'ensemble des structures étatiques. Le dédain manifesté à l'égard de toutes les nouvelles formes de divertissement peut donc être perçu comme un réflexe de défense en provenance de la culture traditionnelle - faite par les hommes, pour un monde gouverné par les hommes - et dirigé contre cette concurrence surgissant d'«en bas» qui propose une alternative "féminine» dérangeant la hiérarchie des rapports de sexes, et donc la domination masculine. Ainsi, jusqu'aux années 60, les controverses sur la culture de masse s'explicitent en termes d'opposition entre le masculin et le féminin, le supérieur et l'inférieur, l'instructif et le distractif, l'actif et le passif 28. Or c'est précisément durant les années 50 que la connotation féminine de la culture populaire est transférée à la télévision qui devient un objet de consommation typiquement féminin, non seulement parce qu'il est acquis par les femmes avec la bourse du ménage, mais également parce qu'il s'adresse aux femmes au foyer formant une majorité du public jouissant, par ailleurs, d'un grand pouvoir d'achat ${ }^{29}$. Il n'est donc pas indifférent de constater que les discours hostiles à l'égard de la télévision, tout comme certains films hollywoodiens manifestant un agacement notoire à l'égard d'un rival économique, mettent en évidence le pouvoir dévastateur d'un médium qui féminise les individus, c'est-àdire qui les rend à la fois passifs, oisifs, frivoles et bêtes.

\section{Images de femmes: la publicité et les soap opera des années 50}

Un aperçu synthétique des recherches portant sur les programmes télévisés des années 50 permet d'éprouver la pertinence de ces thèses concernant la féminisation des téléspectateurs, ainsi que la force du modèle patriarcal qui confine la femme au milieu domestique ${ }^{30}$. Si la rhétorique d'après-guerre invoque la femme au foyer comme symbole et icône de la supériorité des Etats-Unis, dans la réalité sa place au sein de la société s'avère bien plus complexe puisque beaucoup d'entre elles refusent de se plier au modèle dominant en choisissant d'intégrer le marché du travail, au même titre que les hommes. Souvent tiraillées entre les idéaux domestiques et l'ambition d'un développement personnel, les femmes américaines présentent des contradictions qui rendent difficile 
leur réduction à une seule catégorie. L'image de la femme s'occupant uniquement de sa maison et de ses enfants est donc en partie un mythe que la télévision va pourtant nourrir, notamment à travers la publicité qui offre aux téléspectatrices des schémas comportementaux appropriés aux normes morales et sociales. Des études de marché prouvant que le public se différencie en fonction des heures de la journée - féminin le jour, masculin le soir - les annonceurs choisissent de sponsoriser les programmes les plus lucratifs, c'est-à-dire les programmes regardés par les femmes qui travaillent à la maison pour leur famille. La différence sexuelle détermine ainsi la teneur des contenus télévisuels qui sont distribués selon des créneaux horaires jugés plus rentables (séries dramatiques adressées aux femmes) ou plus respectables (informations ou débats politiques adressés aux hommes). Pourtant un problème se pose à ceux qui réfléchissent alors au rôle de la télévision : comment une bonne mère et ménagère trouve-t-elle le temps de regarder la télévision ? $\mathbf{3 1}$

Cette question va beaucoup influencer le développement des premiers soap opera qui se constituent en un genre saturé de contradictions et d'ambiguïtés, redoublant ainsi celles qui sont au cœur de la vie des femmes américaines des années 50. Non seulement une minorité de séries TV joue avec les conventions sociales admises en mettant en scène des personnages féminins engagés dans la vie active et professionnelle, mais les soap les plus populaires explorent en priorité les difficultés liées au mariage et à la parentèle, ce qui vient contredire les stéréotypes de la famille unie et heureuse de l'être. Focalisés sur les relations affectives, ces scénarios dessinent une image contrastée de la famille qui ne fonctionne pas comme une structure stable, car continuellement déchirée par des vengeances, des adultères, des malentendus, des trahisons, des fausses paternités, des reniements, des incestes et même des crimes. Ainsi, les soap opera incarnent une double menace pour la droiture morale de la femme au foyer: d'une part, ils risquent de la distraire de sa mission en tant que citoyenne américaine ouvrant pour le bien de sa famille, et donc de la nation toute entière; d'autre part, ils instillent le virus de la décadence des mœurs en véhiculant l'image de modèles familiaux déviants.

Les producteurs et, parallèlement, les annonceurs, trouveront une parade efficace à ces inconvénients en introduisant des personnages et des modes d'adresse masculins censés conseiller, admonester, éduquer et guider les femmes dans les méandres des déboires familiaux. En effet, les soap opera attribuent toujours aux hommes des rôles de prêtres, de juges, de médecins ou de savants chargés d'énoncer les normes de la bonne conduite en vertu de leurs connaissances et de leur sagesse ${ }^{\mathbf{3 2}}$. Les
31 Irna Philipps, connue pour avoir créé les séries TV et radiophoniques américaines les plus célèbres depuis les années 30 jusqu'aux années 70 , se défendait de distraire les femmes de leur travail domestique car ces programmes n'exigeaient pas une attention visuelle constante, un contact auditif suffisant aussi parfois à suivre l'intrigue si la ménagère avait à s'absenter provisoirement.

32 Ce qui est mis en exergue dans ces séries c'est surtout le fossé qui sépare l'homme et la femme en termes d'autonomie dans un monde fonctionnant selon un système de règles et de valeurs patriarcales. Alors que les hommes recourent, pour comprendre le monde, à des données qui sont celles de la culture masculine dominante (la loi, la technologie, la science, etc.), les femmes, elles, sont représentées comme des individus à la merci d'un savoir dont elles n'ont pas la maîtrise puisqu'elles sont reléguées à la sphère affective et familiale. En désignant les femmes comme étrangères aux valeurs masculines, comme exerçant un mode de compréhension des choses basé sur l'intuition ou l'affect, la télévision ne fait que renforcer la différence sexuelle déjà présente dans la réalité quotidienne. 
33 Ce ton moraliste et moralisant s'estompera dans les années 60 avec l'émergence des mouvements féministes qui obligent l'industrie télévisuelle à adapter son discours à cette nouvelle donne. Les femmes seront alors décrites comme étant elles-mêmes des expertes à l'intérieur de leur maison. La médiation masculine sera écartée au profit d'un mode d'adresse directe à la femme concernée par les produits dont on vante les mérites. Grâce à l'éviction de la voix de l'annonceur, le public peut alors interpréter le message publicitaire à sa guise. De plus, le travail domestique n'est plus montré comme un passe-temps agréable que la femme assume avec joie, mais comme un véritable labeur, à l'instar du travail de son mari. La publicité contribue dès lors à donner de la femme une image valorisante d'elle-même, car elle seule a désormais la maîtrise de son environnement. Mais si la publicité lui confère un sentiment de pouvoir au sein du système familial, elle l'encourage également à participer activement à la régulation de la différence sexuelle.

34 Je rappelle au passage que cette question de l'opposition entre une forme de savoir masculine (rationnelle, déductive) et féminine (intuitive, inductive) est tout à fait centrale dans Fenêtre sur cour où les femmes finissent par résoudre l'énigme grâce à leurs compétences spécifiques.

35 Cf. Elise Lemine, "Voyeurism and the Postwar Crisis of Masculinity in Rear Window", Alfred Hitchcock's Rear Window, John Belton (éd.), op. cit., p. 57-90.

36 Laura Mulvey s'est notamment appuyée sur Fenêtre sur cour pour démontrer comment, dans le cinéma classique hollywoodien, la femme est réduite au statut d'objet du regard masculin. Cf. Laura Mulvey, Visual and other Pleasures, Macmillan, Londres, 1989. A propos de Hitchcock et la théorie féministe, lire Tania Modleski, Hitchcock et la théorie féministe. Les femmes qui en savaient trop, op. cit. Selon elle, le cinéma de Hitchcock ne constitue pas un exemple représentatif du cinéma patriarcal auquel s'attaquent certaines critiques féministes car il fait fonctionner de manière très ambivalente des figures féminines qui peuvent être à la fois sujet et objet du regard. premières séries dramatiques montrent donc des hommes responsables, sachant résoudre les problèmes de leurs enfants et conseiller leur femme dépeinte souvent comme un être incompétent et irrationnel dans le cadre de sa fonction privée. Si, pendant l'épisode, une voix off masculine accompagne et oriente régulièrement le téléspectateur dans son interprétation des événements, la publicité obéit aux mêmes impératifs didactiques et idéologiques. En effet, les annonces publicitaires abondent en médecins, docteurs et autres scientifiques distribuant maints conseils sur la manière de maintenir à la fois la santé familiale et la propreté de la maison, puisque les compétences de la femme au foyer sont encore sujettes à caution ${ }^{33}$. Ainsi, dans les séries TV comme dans la publicité, les femmes apparaissent comme dépendantes d'une autorité patriarcale qui a pour objet d'endiguer les velléités féminines fondées sur un système de valeurs en décalage avec les formes masculines de savoir ${ }^{34}$.

\section{Fenêtre sur cour, le renversement des rôles sexuels et le téléspectateur de télé-réalité}

Si l'on revient à Jeff et à l'hypothèse postulant son statut de téléspectateur, on s'aperçoit que le film de Hitchcock s'inscrit de manière à la fois évidente et subtile dans cette dynamique des genres à l'œuvre dans les années 50. Fenêtre sur cour représente en fait une version originale des contradictions générées par un modèle idéologique phallocentré. Car non seulement l'idéal d'une existence en banlieue, dans une maison remplie de gadgets ultramodernes manipulés par une femme accueillante et serviable, est remplacé dans le film par une variante urbaine du mariage composé par une femme geignarde à souhait, flanquée d'un mari adultère et meurtrier en puissance. Mais aussi parce que le film distribue les rôles sexuels sur un mode peu orthodoxe, Lisa apparaissant comme une figure éminemment masculine, alors que Jeff assume lui une position plutôt féminine au sein de leur couple ${ }^{35}$. En effet, Lisa exhibe plusieurs attributs masculins ou virils pour l'époque: c'est une femme indépendante qui travaille dans le monde de la mode (comme mannequin mais aussi comme femme d'affaires), qui n'aime pas cuisiner (elle apporte des plats préparés par un traiteur), qui prend les initiatives en matière de sexualité (elle intimide aussi bien Jeff que le détective Doyle) et qui va finir (avec Stella, l'infirmière) par maîtriser l'enquête et prendre progressivement le contrôle du regard. Lisa est donc montrée, contrairement aux lectures féministes traditionnelles, comme une femme de caractère, forte d'un pouvoir intellectuel et sexuel qui tranche avec l'image canonique de la femme au foyer véhiculée dans les médias et la publicité $\mathbf{3 6}$. 
De l'autre côté, le rôle de Jeff permet d'avoir accès au double caché du modèle de la mère de famille: l'homme qui ne correspond pas à l'idéal masculin des années 50, hérité de la Seconde Guerre mondiale et fondé sur les valeurs de courage, de liberté et d'esprit aventurier (fig. 11 et 12), s'expose au danger d'une féminisation. Rappelons que Jeff est un ancien soldat qui rêve de partir vers des contrées lointaines et sauvages (les destinations de Shangaï, Brésil, Hong Kong, l’Himalaya sont évoquées tour à tour), tout comme nombre d'hommes de cette génération qui rejettent le modèle petit-bourgeois de l'employé de bureau ou du cadre supérieur, au profit d'une vie à l'air libre, telle qu'elle est vantée dans les westerns de l'époque ${ }^{37}$. Ainsi, emprisonné et immobilisé à la maison, Jeff se retrouve exactement dans la même situation que la majorité des femmes américaines qui réservent leurs activités à l'espace domestique et qui, pour se distraire, regardent la télévision. Alors qu'avant son accident, Jeff n'aurait probablement jamais songé à observer ses voisins, sa paralysie provisoire l'oblige à passer des journées entières chez lui, l'encourageant du même coup à adopter un comportement habituellement associé à la femme au foyer. Il devient, à l'instar du prototype de la téléspectatrice construit par le discours social, un être passif et paresseux, qui délaisse son devoir premier pour se plonger, avec une curiosité aussi malsaine que féminine, dans le secret de la vie d'autrui 38 .

Dans le film, ce surinvestissement de la fonction scopique permet d'activer trois sens ou champs d'activité de la notion de voyeurisme, sens qui sont par ailleurs tous référés à la féminité. Si le voyeurisme est d'abord, au sens clinique du terme, une pathologie mentale et sexuelle, il est également compris, dans le langage courant, comme une perversion fort répandue dans une société contemporaine volontiers encline à pénétrer dans la sphère intime des gens. Plus précisément, cette notion s'applique aux consommateurs des médias de masse comme la presse people (dont le lectorat est majoritairement féminin), et surtout la télévision. En 1954, Jeff incarne ces trois formes de voyeurisme: investi totalement dans son "enquête", il souffre d'une perversion sexuelle passive qui le féminise et l'oppose à Lisa qui, elle, affiche une sexualité active; de plus, il s'intéresse secrètement à la vie privée des autres, assumant un défaut - la curiosité - typiquement féminin ; enfin, il se glisse dans la peau d'un homme qui tente d'oublier sa désagréable condition en regardant des images animées en vis-à-vis, tout comme la ménagère qui appréhende la télévision comme un moyen d'évasion. Il se voit donc triplement féminisé. En construisant des images de femmes et d'hommes tout à fait ambivalentes, Hitchcock joue admirablement avec les frontières de genre que l'Amérique s'est fixée dans les années 50. Si
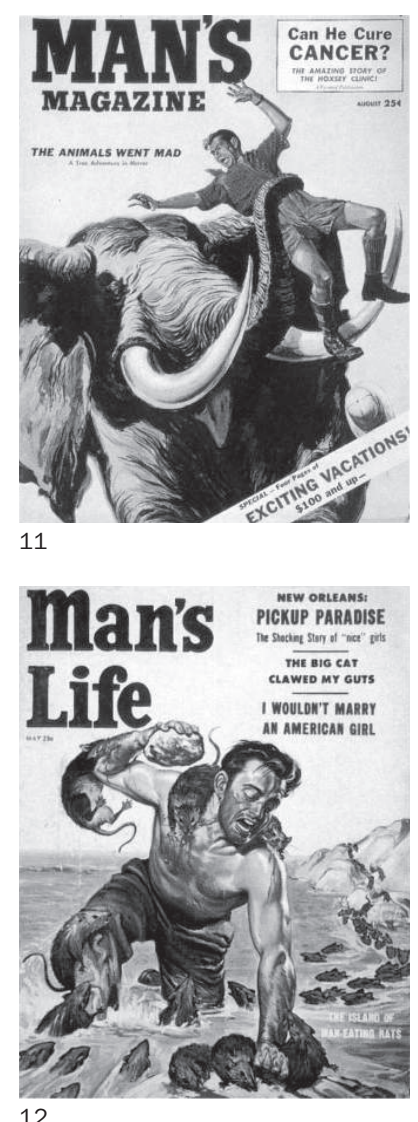

37 John Wayne incarne d'ailleurs un modèle masculin très admiré par le public des années 50. Le genre du western permet en effet aux anciens soldats qui ont cru en leur fonction de protecteurs de la nation de retrouver, à la fin de la guerre, par le biais d'une identification fantasmatique, un peu de cette virilité et de cette fierté perdues en raison de leur retour à la "civilisation", les éloignant ainsi définitivement de la griserie des champs de bataille. Jeff préfère donc ce modèle à celui qui lui est présenté via les images de couples entraperçues au-delà de la cour, celles des Thorwald servant de repoussoir absolu.

38 Jeff n'est cependant pas totalement passif face à ces fenêtres-écrans qui n'appellent pas uniquement une pure contemplation du perçu (la danse chaloupée de Mlle Torso), mais qui l'incitent aussi à se lancer dans une enquête criminelle, au prix d'une deuxième jambe cassée. 
Jeff répond, à certains égards, à l'idéal masculin de l'homme libre multipliant les conquêtes, il apparaît aussi comme un homme dont la virilité est remise en question sur différents plans. Son personnage permet donc de problématiser, d'une part, les rapports de sexe dont les différentes lignes narratives font état, d'autre part, d'incarner un sujet-percevant indexé autant par un discours social féminisant la télévision que par cette boîte à images destinée à dominer la culture de masse.

En tenant compte du contexte culturel et social dans lequel Fenêtre sur cour est né, ainsi que de l'importance croissante prise par la télévision dans le monde occidental, il n'est peut-être pas totalement déplacé d'avancer que le personnage de Jeff préfigure en quelque sorte le téléspectateur actuel (féminin aussi bien que masculin), avide d'aventures humaines et d'émotions fortes fournies, en particulier, par les programmes de télé-réalité. Cette hypothèse se trouve confortée si l'on admet que notre société actuelle repose sur des bases qui ont été jetées par cette Amérique des années 50, et qu'une grande partie de l'idéologie des mass media nous a été transmise par les Etats-Unis, avec le temps de retard, cependant, qui sied si bien aux Européens. Le film de Hitchcock parvient, ainsi, à articuler une définition culturelle de la télévision aussi inattendue que pertinente au vu de son évolution ultérieure. En 1955, les rapports entre Hitchcock et la télévision passeront de l'implicite à l'explicite à l'occasion de la signature d'un contrat avec la M.C.A pour une série de téléfilms intitulée Alfred Hitchcock Presents. Il réalisera ainsi, de 1955 à 1962, 22 films pour la télévision.

CEUVRE D'UN JEUNE ARTISTE AMÉRICAIN CONTEMPORAIN, RYAN JOHNSTON, QUI TÉMOIGNE DE LA PRÉGNANCE ET DE LA PÉRENNITÉ DE L'IMAGERIE DES ANNÉES 50, TOUT EN ARTICULANT DES THĖMES ABORDÉS DANS CET ARTICLE: L'AMÉRIQUE, LE CINÉMA, LA FEMME AU FOYER ET LA SOCIÉTÉ DE CONSOMMATION

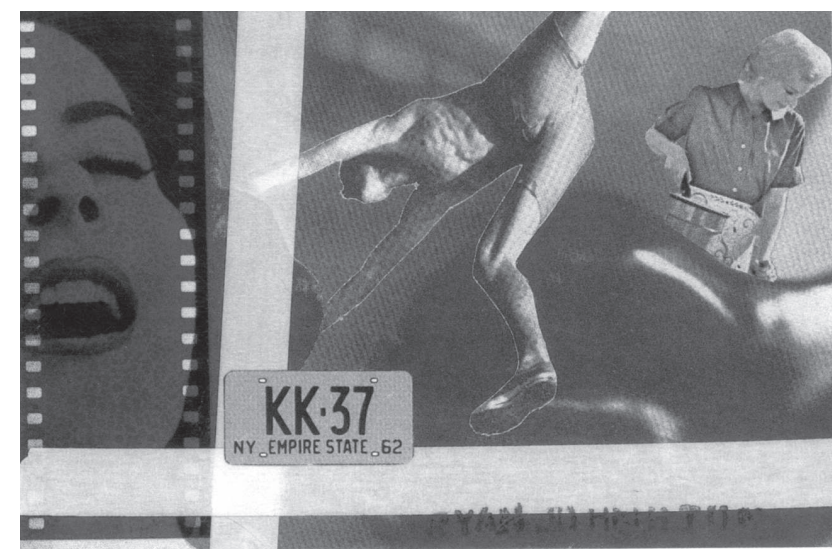

